\title{
OCULAR INJURIES IN MID-FACIAL FRACTURES: A RETROSPECTIVE STUDY
}

\author{
Hussein Hatem *, Khaled Amr ${ }^{* *}$ and Omnia I. Sultan ${ }^{* * *}$
}

\begin{abstract}
Purpose: Mid-face injuries are usually accompanied with loss of integrity of the orbital skeleton along with the subsequent affection of the visual apparatus. This study aimed to highlight the incidence of different eye injuries associated with mid-facial fractures and to accentuate the need of the maxillofacial surgeon to comprehend and approach the post-traumatic ophthalmic consequences.
\end{abstract}

Patients and methods: A total of twenty five patients suffering from mid-facial fractures and orbital involvement were included in this study from the departments of oral and maxillofacial surgery, faculties of Dentistry, Cairo University and MSA University. They were 22 males and 3 females with a mean age of 37 years. All the patients were subjected to immediate clinical and radiographic examination to evaluate the extent of the injury and its implications. An ophthalmologist was asked to further assess the eye apparatus to determine the exact ophthalmic complications. The collected clinical, radiographic and ophthalmic findings were recorded and analyzed.

Results: A dominating etiology of Road Traffic Accidents was found (64\%) with a significant male predominance $(88 \%)$. Mid-face trauma in the form of ZMC fracture was acquainted as the most common reason for ophthalmic problems $(36 \%)$ followed by the direct orbital blow-out fractures $(20 \%)$. A wide range of ophthalmic complications were recorded. Sub-conjunctival hemorrhage (92\%) was the most common to occur followed by peri-orbital edema (84\%) and lid ecchymosis and laceration (76\%). A clinical complaint from sensory infra-orbital nerve paresthesia was documented (72\%). Diplopia (48\%) and Enophthalmus (24\%) were encountered as the most severe types of complications with other non-significant occurrence of reduced vision (8\%), retinal detachment (4\%), corneal problems (8\%) and traumatic hyphema (8\%).

Conclusion: We concluded that it is a must to extend the maxillofacial surgeon's knowledge to include post-traumatic ocular injuries and their ophthalmic complications to complement an optimal patients' treatment outcome and to prevent disastrous ophthalmic repercussions following mid-face injuries.

KEYWORDS: Mid-face Fracture, ocular injuries, ophthalmic complications.

* Associate Professor of Oral and Maxillofacial Surgery, Oral and Maxillofacial Surgery Department, Faculty of Dentistry, Cairo University.

** Lecturer of Oral and Maxillofacial Surgery, Oral and Maxillofacial Surgery Department, Faculty of Dentistry, Cairo University.

*** Lecturer of Oral and Maxillofacial Surgery, Oral and Maxillofacial Surgery Department, Faculty of Dentistry, October University of Modern Sciences and Arts (MSA). 


\section{INTRODUCTION}

Orbital fracture usually occurs in patients presented with mid-facial trauma. Mid-face injuries responsible for ocular complications mainly included zygomatico-maxillary complex fractures, Le Forte II and III fractures and pure blow out fractures of the orbit ${ }^{(1-2)}$.

The eye apparatus responsible for a vital role; vision, is naturally protected by the hard bony orbital rim and the soft orbital fat and muscles ${ }^{(3)}$. Despite this dual protection system, mid-face injuries accommodate for $68-95 \%$ ocular injuries with associated ophthalmic complications accounting for 19 million blind or reduced vision people worldwide $^{(4)}$.

Several ocular injuries were reported in the literature ${ }^{(5-6)}$. Ophthalmic complications ranged from clearly apparent to the surgeon to hidden potentially dangerous complications which need meticulous examination. Thus, improper assessment may compromise the patient's vision with its consequent medico-legal repercussions.

These injuries may vary from mild subconjunctival hemorrhage to major globe rupture and loss of vision. This depends mainly on the type, site and magnitude of the incoming trauma. Although blindness is uncommon, it has been documented in the literature ${ }^{(5,7)}$.

Authors stated that repairing the fractures by surgery before the ophthalmic correction might further damage the visual outcome. Hence, thorough assessment and correction of the eye is mandatory before and after the surgical correction of the fracture ${ }^{(8)}$.

In this study, in an attempt to reach a more efficient management of the mid-face trauma and to prevent disastrous ophthalmic complications, ophthalmologists were integrated in the team in the process to diagnose missed post-traumatic complications.

\section{PATIENTS AND METHODS}

Twenty five patients were incorporatedin this studyfrom the departments of oral and maxillofacial surgery, Faculties of Dentistry, Cairo University and MSA University. They all had a displaced midfacial fracture with orbital skeleton involvement which required surgical interference. An ethical clearance and a written consent were obtained from each patient.

\section{Exclusion criteria:}

- History of a pre-existing ophthalmic diseases or ocular disorders as glaucoma or retinal disorders.

- Infection and/or systemic diseases as Diabetes Mellitus.

\section{Preoperative assessment:}

History, Gender and cause of the trauma were recorded. The patients had a mean age of 37 years.

Immediate clinical examination was performed to evaluate the extent of the injury and its implications. The evaluation for ocular injuries included testing the ocular motility, papillary reaction to light and assessment of the sensory infra-orbital nerve.

The eye was further examined by an ophthalmologist to look for additional ophthalmic complications. Various tests were performed including the Snellen chart test for Visual acuity. Advanced diagnostic tests were applied as Retinoscopy, fundoscopy, fluorescent staining,slit lamp examination, Schiotz tonometer, and Hertelexophthalmo-meter. The findings of the maxillofacial surgeon and the ophthalmologist were documented.

Preoperative CT images were requestedto identify the exact fracture sites and their effects on the eye. The collected clinical, radiographic and ophthalmic findings were recorded and analyzed. 


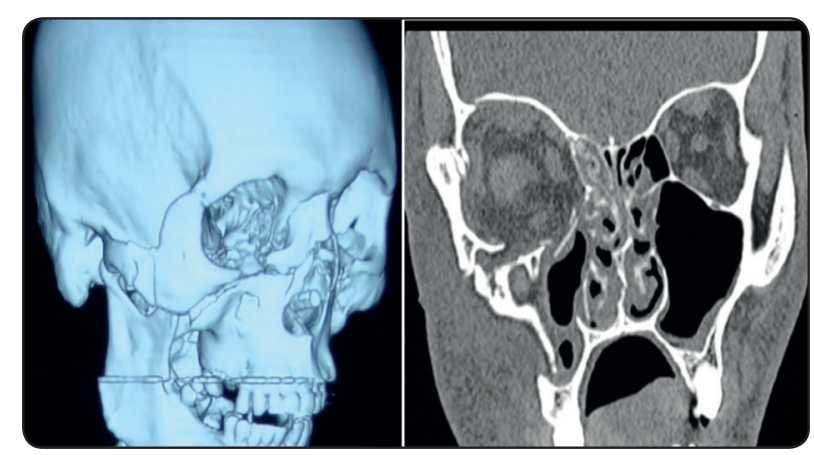

Fig. (1) CT images showing mid-face fractures associated with orbital fracture.

\section{RESULTS}

A dominating etiology of Road Traffic Accidents (RTA) (64\%) was found. Nine cases only (36\%) were due to inter-personal violence. A male predominance of $88 \%$ (22 cases) was documented in opposition to only 3 female cases (12\%).

Various types of mid-facial trauma were reported with the disruption of the ocular skeleton affecting the eye apparatus.ZMC fracture was acquainted for the most common reason for ophthalmic problems $(36 \%)$. This was followed by the direct orbital blow-out fractures (20\%). Fracture of the orbital rim (4\%), Le Fort fractures (12\%) and combination of the previously mentioned fractures (28\%) were also found. (Table 1)

TABLE (1) presenting the incidence of mid-face fracture type.

\begin{tabular}{|c|l|c|c|}
\hline & \multicolumn{1}{|c|}{ Type } & Number & Percentage \\
\hline 1 & ZMC fractures & 9 & $36 \%$ \\
\hline 2 & Pure blow out orbital fractures & 5 & $20 \%$ \\
\hline 3 & Fracture of the orbital rim & 1 & $4 \%$ \\
\hline 4 & Le Fort fractures & 3 & $12 \%$ \\
\hline 5 & Combination & 7 & $28 \%$ \\
\hline
\end{tabular}

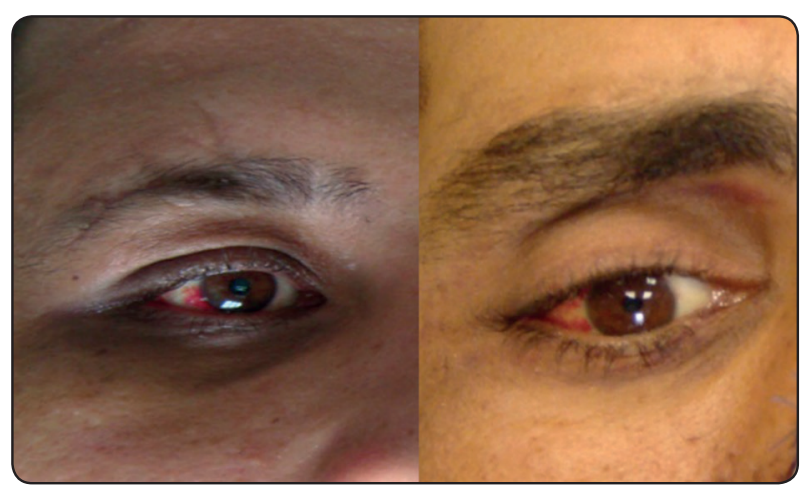

Fig. (2) Photographs showing sub-conjunctival hemorrhage.

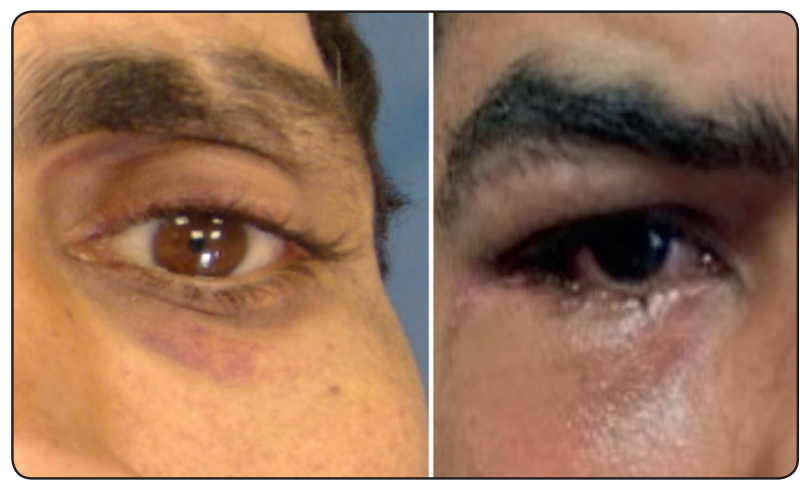

Fig. (3) Photographs showing lid edema and peri-orbital hematoma.

Blunt injuries of the globe were found in most of the cases $(96 \%)$. Only one patient was presented with a penetrating globe injury $(4 \%)$.

Various ophthalmic complications were recorded. Sub-conjunctival hemorrhage (92\%) was the most common to occur. While peri-orbital edemaor hematoma (84\%), lid ecchymosis and laceration (76\%)appeared to be the next commonfindings in most of the patients.

Eighteen patients (72\%) complained from sensory infra-orbital nerve paresthesia. This was confirmed clinically with the two-point discrimination test.

Diplopia (48\%) and Enophthalmus (24\%) were encountered as the most severe types of complications. (Fig 4) 


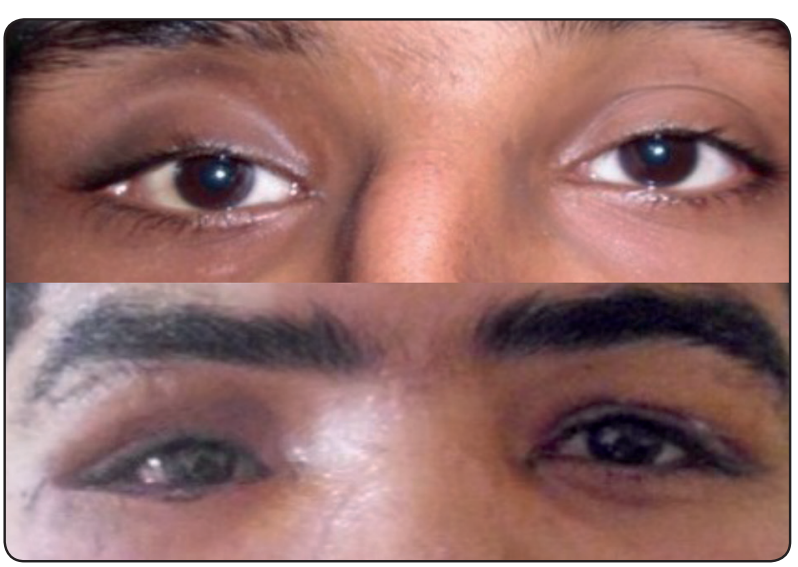

Fig. (4) Photographs showing Enophthalmus.

Other complications as reduced vision (8\%), retinal detachment (4\%), corneal problems (8\%) and traumatic hyphema $(8 \%)$ appeared non-significant in this study.

Along the entire study, the ophthalmologist further diagnosed minor complications missed by our (maxillofacial surgeon's) examination in eight cases (32\%). These included corneal abrasion, lens subluxation and retinal detachment.(Table 2) (Fig 5)

TABLE (2) presenting the detected types of ocular injuries.

\begin{tabular}{|l|l|l|l|}
\hline 1 & Blunt injury & 9 & Pain on ocular \\
3 & Penetrating injury & & movements \\
& Sub-conjunctival hemor- & 10 & Diplopia \\
4 & rhage & 11 & Enophthalmus \\
& toma & 12 & Retinal detachment \\
5 & Lid ecchymosisor laceration & 14 & Reduced vision \\
6 & Infra-orbital nerve pares- & 15 & Retro-bulbar hemor- \\
7 & thesia & 16 & rhage Vitreous hem- \\
8 & Corneal abrasion or edema & Proptosis & \\
\hline
\end{tabular}

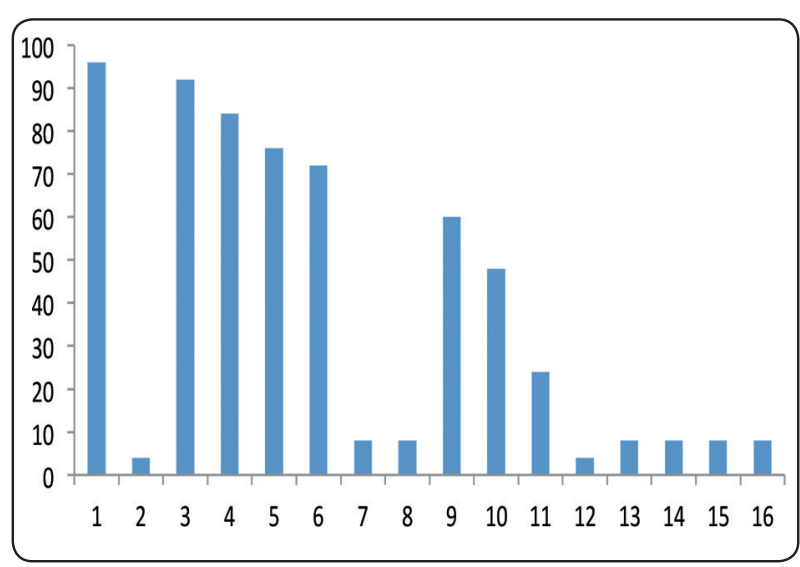

Fig. (5) A bar chart showing the incidenceof ocular injuriesin percentage.

\section{DISCUSSION}

Many studies were performed in attempt to link mid-face trauma to ocular injuries and their subsequent ophthalmic complications.

Different occurring statistics were recorded due to the non-standardized minor and major ophthalmic complications incorporated in the literature.

In this study, similar to Magarakis ${ }^{(6)}$, ZMC fractureswere responsible for most of the ophthalmic problems $(36 \%)$ followed by the direct orbital blowout fractures $(20 \%)$.

A male predominance of $88 \%$ was found with a high incidence cause of Road Traffic Accidents (64\%). This came in accordance with Patil et al $\boldsymbol{l}^{(3)}$ and nabeela et $\boldsymbol{a l}^{(9)}$, which was normal considering the fact that a higher number of males are driving the vehicles than females. The second highest recognized cause was the inter-personal violence (36\%); comparable to what was declared by Roccia et al ${ }^{(10)}$.

Various ophthalmic complications have been reported to occur due to ocular injuries associated with mid-face fractures, hence the lack of uniform classification in the literature.

However, authors agreed upon the necessity for rapid clinical assessment and interference to avoid further damage and visual compromise ${ }^{(7,11)}$. 
The results of this study stated that sub-conjunctivalhemorrhage was the most common occurring ophthalmic problem (92\%) which was similar to Mittal et al ${ }^{(12)}$ and Wilson ${ }^{(13)}$ who agreed on its spontaneous resolution without any interference.

A high incidence of peri-orbital edema or hematoma (84\%) and lid ecchymosis and laceration (76\%) was recorded, comparable to all studies, which was normal to find due to the loose connective tissue around the eye. They are the first signs of trauma which in turn made the clinical examination difficult ${ }^{(3,11)}$.

Eighteen patients (72\%) complained from sensory paresthesia of the infra-orbital nerve. This was due to the impingement of the displaced fractured segments on the supplying nerves.

Major complications as Diplopia (48\%) and Enophthalmus (24\%) were encountered in this study.

Diplopia due to edema resolved spontaneously within 7-10 days. However, Diplopia persistence due to ocular muscle entrapment or suspensory ligament attachment displacement varied according to the presented type and location of orbital fracture, and so, needed surgical interference and correction ${ }^{(14-15)}$.

On the other hand, Enophthalmos was due to the increase of the skeletal orbital volume after fracture. Hence, after resolution of the edema and hematoma, Whitehouse et al ${ }^{(16)}$ stated that every $1 \mathrm{~cm}$ increase of the skeletal orbital volume encountered $0.8 \mathrm{~mm}$ Enophthalmos.

In this study, non-significant number of other complications as reduced vision (8\%), retinal detachment $(4 \%)$, corneal problems $(8 \%)$ and traumatic hyphema $(8 \%)$ were recorded. This was analogous to Rajkumar et al ${ }^{(4)}$ and Foroughi et al ${ }^{(7)}$ who, nevertheless, stressed on the importance to address them and the morbidity of neglecting such complications.

Some ophthalmic injuries (32\%) could have left undetected if not for the ophthalmologist's experience and specialized equipment. Unfortunately, the maxillofacial surgeons' knowledge nowadays does not level up to explore hidden ophthalmic complications as an ophthalmologist. Hence, the integration of an ophthalmologist in the mid-face trauma team is mandatory.

\section{CONCLUSION AND SIGNIFICANCE}

This study delineated the need to extend the maxillofacial surgeon's comprehension of ophthalmic injuries in mid-face trauma patients to complement an optimal patients' treatment.

Such knowledge render the maxillofacial surgeon the experience in preventing missed ophthalmic complications and deciding the optimum timing for surgical intervention as some ocular injuries are time-sensitive.

Detailed preoperative ophthalmic tests' results should be documented for comparison of pre and post-surgical visual status for medico-legal concern.

Further studies concerning the relation between specific ocular complications to specific trauma pattern is recommended.

Most of the complications were fracture-dependant which resolved after surgical interference. The need to correlate pre and post surgical ophthalmic findings is evident.

\section{REFERENCES}

1. Al-Qurainy IA, Stassen LF, Dutton GN et al. The characteristics of mid-facial fractures and the association with ocular injury: A prospective study. Br J Oral Maxillofac Surg; 29: 291,1991.

2. Riaz N, Chatha AA, Warraich RA et al. Ophthalmic injuries in orbito-zygomatic fractures. $\mathrm{J}$ of the college of Physicians and Surgeons; 24: 649, 2014.

3. Patil SG, Kotwal IA, Joshi U et al. Ophthalmic evaluation by a maxillofacial surgeon and an ophthalmologist in assessing the damage to the orbital contents in mid-facial fractures: A prospective study. J Maxillofac Oral Surg; 15: 328, 2016. 
4. Rajkumar GC, Ashwin DP and Rudresh KB. Ocular injuries associated with mid-face fractures: A 5 year survey. $\mathrm{J}$ Oral Maxillofac Oral Surg; 14: 925, 2015.

5. Karabekir HS, Gocmen-Mas N, Emel E et al. Ocular and peri-ocular injuries associated with an isolated orbital fracture depending on a blunt cranial trauma: anatomical and surgical aspects. J CraniomaxillofacSurg; 40: 189, 2012.

6. Magarakis M, Mundinger GS, Kelamis JA et al. Ocular injury, visual impairment and blindness associated with facial fractures: a systemic literature review. PlastReconstrSurg; 129: 227, 2012.

7. Foroughi R, Faghani F, Gharib S et al. Traumatic ophthalmic injuries in mid-face trauma: A decade long survey. $\mathrm{J}$ Craniomax Res; 2: 128, 2015.

8. Jamal BT, Pfahler SM, Lane KA et al. Ophthalmic injuries in patients with zygomatico-maxillary complex fractures requiring surgical repair. J Oral Maxillofac Surg; 67: 986, 2009.

9. Nabeela R, Asad AC, Riaz AW et al. Ophthalmic injuries in orbito-zygomaticfractures. J CollPhysSurg Pak; 24: 649, 2014.
10. Roccia F, Boffano P, Guglielmi V et al. Role of the maxillofacial surgeon in the management of severe ocular injuries after maxillofacial fractures. J Emerg Trauma Shock; 4: $188,2011$.

11. Board SP, Linden J. Trauma to the globe and orbit. Emerg Med Clin N Am; 26: 97, 2008.

12. Mittal G, Singh N and Mittal SR. A prospective study on ophthalmic injuries related to maxillofacial trauma in Indian population. Natl J MaxillofacSurg; 3: 152, 2012.

13. Wilson RJ. Sub-conjunctival hemorrhage: overview and management. J Am OptomAssoc; 57: 376, 1986.

14. Folkestad L, Lindgren G, Moller C et al. Diplopia in orbital fractures: A simple method to evaluate eye morbidity. ActaOrolaryngol; 127:156, 2007.

15. Tadj A and Kimble FW. Fractured zygoma. ANZ J Surg; 73: 49, 2003.

16. Whitehouse RW, Batterbury M, Jackson A et al. Prediction of enophthalmos by computed tomography after 'blow out' orbital fracture. Br J Ophthalmol; 78: 618, 1994. 